\title{
Series Production and Test of Hybrid Modules for the ALICE ITS Upgrade
}

\author{
M. Buckland for the ALICE collaboration* \\ University of Liverpool, United Kingdom \\ E-mail: bucklandeliverpool.ac.uk
}

\begin{abstract}
ALICE is one of the four experiments at the LHC located at CERN. As part of the upgrade of the detector, the current Inner Tracking System (ITS) will be replaced by an all silicon detector constructed from pixel sensors with a pitch of $27 \times 29 \mu \mathrm{m}^{2}$ using CMOS Monolithic Active Pixel Sensors (MAPs) technology. The goal of this upgrade is to enable precise measurements of low momenta particles by significantly improving the impact parameter resolution, tracking efficiency and readout capacity. The detector consists of 7 concentric layers split into two barrels, an inner barrel and an outer barrel. To construct a layer in the detector several sensors are arranged and glued to an Flexible Printed Circuit (FPC) and electrically connected through wirebonds to create a hybrid module. These modules are then joined together to make the staves and then finally the barrels that vary in size depending on the layer. The module production was carried out at five assembly sites and has now finished. In total over 2500 modules were produced with a yield of $84 \%$ for detector grade modules. Each module underwent wire pull tests to assess the quality of the bonds, extensive electrical tests to evaluate the functionality of the module and classify it based on the results. Metrology was also carried out on selected modules to better understand the mechanical properties and the quality of the assembly during the construction phase. This proceeding will present the assembly procedure of the modules, give details and results of the tests carried out and a summary of the production as a whole.
\end{abstract}

The 28th International Workshop on Vertex Detectors - Vertex2019

13-18 October, 2019

Lopud, Croatia

${ }^{*}$ Speaker. 


\section{Introduction}

ALICE (A Large Ion Collider Experiment) [1] is one of the four experiments at the Large Hadron Collider (LHC) located at CERN. ALICE is optimised for heavy-ion physics and is dedicated to the investigation of the physics properties of the Quark-Gluon Plasma and new phenomena in QCD. It achieves this by making measurements of ultra-relativistic pp, $\mathrm{p}-\mathrm{Pb}$ and $\mathrm{Pb}-\mathrm{Pb}$ collisions. The LHC is currently at Long Shutdown 2 (2019-2020) where the accelerator and the detectors are undergoing maintenance and upgrades in preparation for Run 3. ALICE will be upgraded with the main goal of high precision measurements of rare processes down to low transverse momentum $\left(p_{T}<1 \mathrm{GeV} / \mathrm{c}\right)$ [2]. One key part of the ALICE upgrade is the upgrade of the Inner Tracking System (ITS) [3].

\section{ALICE Inner Tracking System Upgrade}

One of the requirements for the upgrade is to improve the impact parameter resolution. This will be achieved by moving closer to the Interaction Point (IP), from $39 \mathrm{~mm}$ to $22 \mathrm{~mm}$, with a new beam pipe and reducing both the material budget (from $1.14 \% X_{0}$ to $0.3 \% X_{0}$ ) and pixel size (from $425 \mu \mathrm{m} \times 50 \mu \mathrm{m}$ to $27 \mu \mathrm{m} \times 29 \mu \mathrm{m})$. Another requirement is to improve the tracking efficiency and $p_{T}$ resolution by increasing the granularity from 6 to 7 layers and using pixel sensors. The upgraded ITS is entirely based on Monolithic Active Pixel Sensors (MAPS). Furthermore, the readout rate will need to be increased by a factor of 100. Finally, one feature of the ITS upgrade will be the possibility for removal and insertion during the yearly shutdown. A comparison between the ITS and the ITS upgrade is shown in table 1.

The layout of the ITS upgrade consists of 7 concentric layers split into two barrels, the Inner Barrel (IB) and the Outer Barrel (OB), shown in figure 1. The first 3 layers, closest to the IP, make

Table 1: A table comparing different properties of the ITS to the ITS upgrade.

\begin{tabular}{lcc}
\hline & ITS & ITS Upgrade \\
\hline Technology & Hybrid pixel, strip, & MAPS \\
Max rate $(\mathrm{Pb}-\mathrm{Pb})$ & drift & $100 \mathrm{kHz}$ \\
Thickness & $1 \mathrm{kHz}$ & Inner barrel: $0.3 \% X_{0}$, \\
Pixel size & $1.14 \% X_{0}$ & Outer barrel: $1 \% X_{0}$ \\
Spatial resolution $(\mathrm{r} \phi \times \mathrm{z})$ & $12 \mu \mathrm{m} \times 100 \mu \mathrm{m}$ & $5 \mu \mathrm{m} \times 5 \mu \mathrm{m}$ \\
Raduis & $39-430 \mathrm{~mm}$ & $22-395 \mathrm{~mm}$ \\
Rapidity coverage & $|\eta|<0.9$ & $|\eta|<1.3$ \\
Layers & 6 & 7 \\
Readout & Analog (drift, strip), & Digital \\
& Digital (pixel) & \\
\hline
\end{tabular}




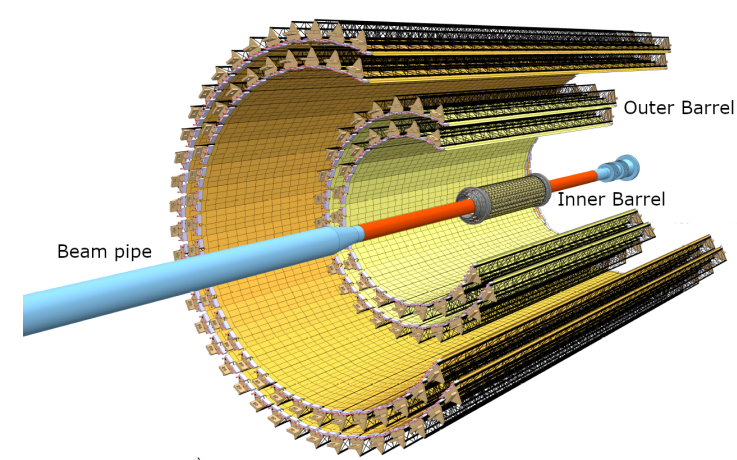

Figure 1: Layout of the ITS upgrade [3].

up the IB with a total of 48 staves, each with 1 module. For the OB, there are 4 layers further split into 2 Middle Layers (ML) and 2 Outer Layers (OL). In the ML, there are a total 54 staves, each with 8 modules and a length of $843 \mathrm{~mm}$. In the OL, there are a total of 90 staves, each with 14 modules and a length of $1475 \mathrm{~mm}$. In the upgraded ITS the total active area of silicon is $10 \mathrm{~m}^{2}$ and it contains 12.5 billion pixels.

\section{Module Design and Assembly}

The module design is to glue chips to a Flexible Printed Circuit (FPC) then electrically connect the two together with wirebonds to form a Hybrid Integrated Circuit (HIC). In the IB, 9 ALPIDE $[3,4]$ chips $(50 \mu \mathrm{m}$ thick) are glued to an aluminium FPC and the wirebonds transfer the control, clock, data and power. Figure 2a shows a picture of the sensor side of an IB module. The chips are read out separately due to the high hit density expected in the IB, $>50 \mathrm{~cm}^{-2}$ in central $\mathrm{Pb}$ $\mathrm{Pb}$ collisions. The IB module production all took place at CERN. For the OB, 14 ALPIDE chips (100 $\mu \mathrm{m}$ thick) are glued to a copper FPC in two rows of 7. The wirebonds transfer the control, clock and data, while the power is delivered via 6 cross-cables soldered to the FPC. In the OB module the data and control are transferred through 1 master chip per row due to the lower hit

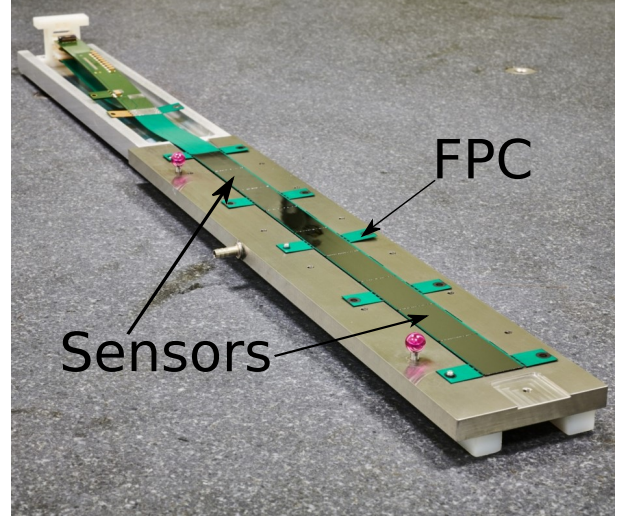

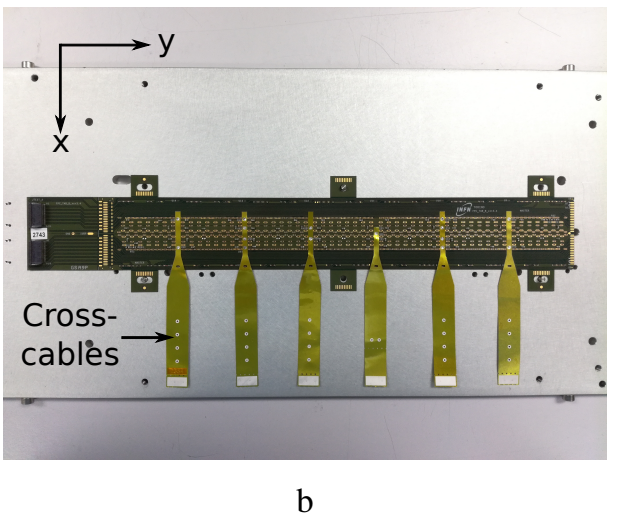

b

Figure 2: (a) The sensor side of an inner barrel module. (b) The FPC side of an outer barrel module. 


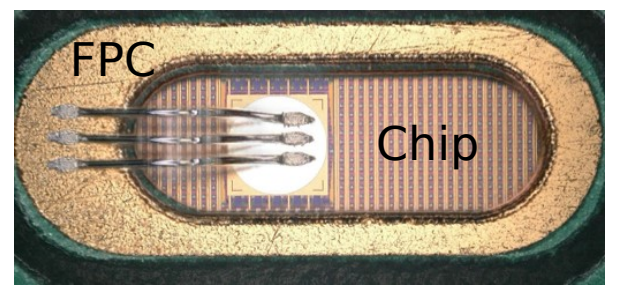

Figure 3: Wirebonds connecting the FPC pad to the chip pad.

density expected in the $\mathrm{OB},<1 \mathrm{~cm}^{-2}$ in central $\mathrm{Pb}-\mathrm{Pb}$ collisions. A picture of an $\mathrm{OB}$ module is shown in figure $2 b$ with the coordinates used in the metrology labelled and the wirebonds are shown in figure 3. The OB module construction was carried out by 5 sites: Bari (IT), Liverpool (UK), Pusan (KR), Strasbourg (FR) and Wuhan (CH).

To assemble the modules a custom designed machine produced by IBS Precision Engineering (NL) and designed to the specifications needed for the project was used, the so-called Module Assembly Machine (MAM) shown in figure 4. The MAM allowed for automatic pick up and placement of the chips to within a $5 \mu \mathrm{m}$ alignment precision. It also included a camera that was able to perform inspections on the chips. These were an edge inspection to see if any cracks on the edge passed through the protective seal rings and also dimension inspections to see if the chips were within tolerance for their width and length. Once the modules are assembled the Quality Assurance (QA) process begins.

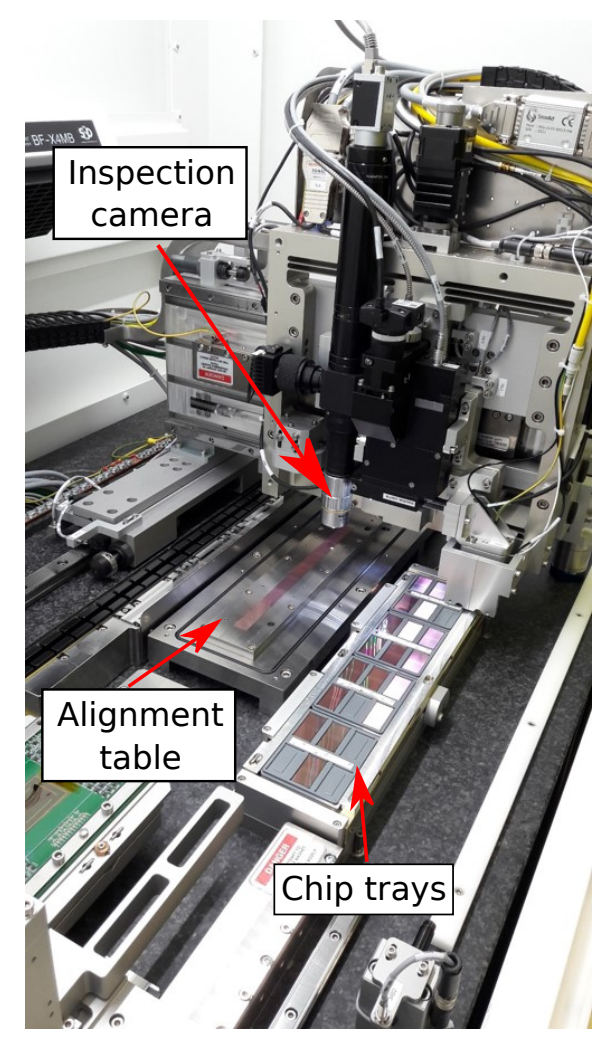

Figure 4: The module assembly machine used to position and inspect the chips. 


\section{Module QA: Functional Tests}

Once the module has been produced, a series of functional tests would be carried out. The setup of the tests is shown in figure 5a. The first test is called the impedance test and is carried out to determine if there is a short on the module. The impedance test does this by measuring the impedance between the power lines (digital, analogue and bias) with respect to the ground. If the impedance was found to be below $10 \Omega$ then a short was declared and a thermal camera would be used to investigate and find any potential source of the short. The next functional test is the single module test. This is a set of scans carried out (e.g. FIFO, threshold and noise) in order to determine the functionality of the readout, analogue and digital circuitry. An example of a threshold scan result is shown in figure 5b. The scans were also carried out at different biasing conditions e.g. supply voltage $\pm 10 \%$, with no back bias supplied and a back bias of $-3 \mathrm{~V}$. The final functional test for the modules was the endurance test. In this test 10 modules were tested at once and undergo 130 power cycles (the expected operation during the lifetime of the detector) to determine the stability of the module by monitoring the supply currents, temperature and FIFO response. The results of all the functional tests were used to classify the modules as detector grade or not.

\section{Module QA: Mechanical Tests}

In addition to the functional tests, there are also mechanical tests. These set of tests were carried out on a select number of modules, e.g. 1 in 10. One such test was the group of metrology test to determine the module thickness, flatness, dimensions and chip-FPC alignment (the coordinates used in the metrology are shown in figure $2 b$ ). The results of these tests would give insights to the mechanical properties and the quality of the assembly during the construction phase. The metrology routines were carried out using a SmartScope and only carried out on OB modules. An example of one of the metrology scans is shown in figure 6a. Another test was the pull test of the wires using a Dage 4000 BondTester. This measures the force needed to remove a bond and records the different bond breaking mechanisms. Since each bond pad has 3 wires, when one wire was removed during the pull test, the module would still be functional and acceptable as detector

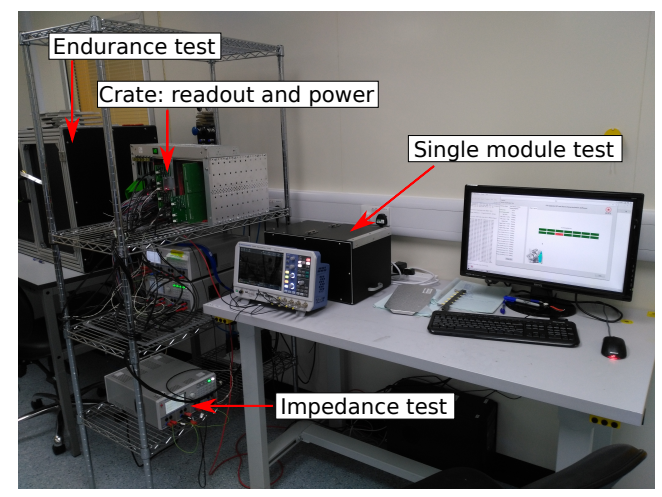

a

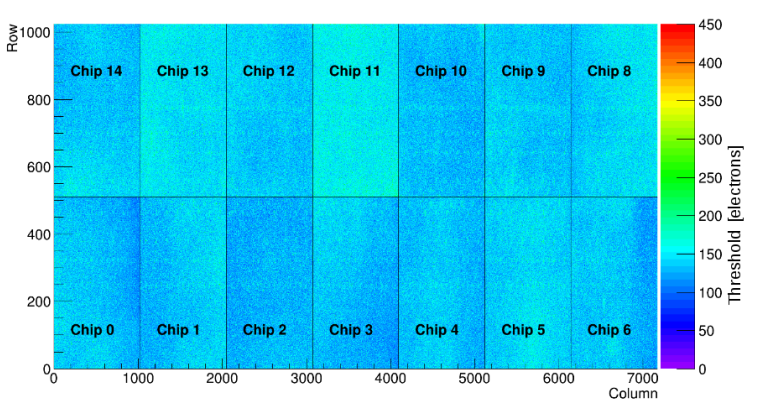

b

Figure 5: (a) The testing stations at Liverpool. (b) An example threshold map for an OB module. 


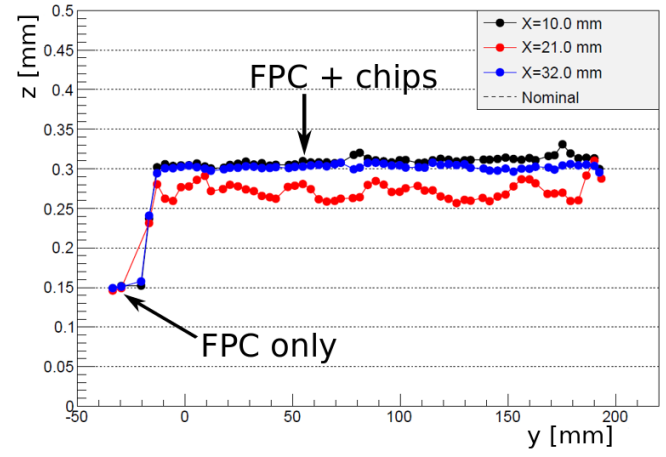

a

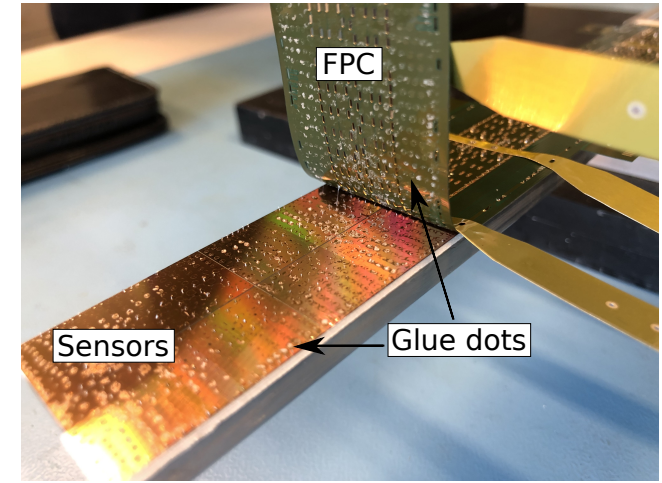

$\mathrm{b}$

Figure 6: (a) An example of the OB module metrology showing the thickness (z) and flatness. (b) A picture of a peel test for an $\mathrm{OB}$ module.

grade. Once a set number of wires on a module was pulled, the results were analysed and determined detector grade if the average force $>10 \mathrm{~g}$, the standard deviation $<1.5 \mathrm{~g}$ and the minimum force $>5 \mathrm{~g}$. The final mechanical test was the peel test that determines the quality of the gluing between the chips and FPC. It does this by measuring the force it takes to peel the FPC away from the chips, as shown in figure 6b. If the average force of the peel test was $>200 \mathrm{~g}$ then the module was deemed to have been glued sufficiently and if it was below this was an indicator that something in the glueing procedure was not correct. The test is destructive so was only carried out on dummy modules or on modules previously classified as not detector grade.

\section{Module Production Summary}

The module production for both the IB and OB has been completed. For the IB, a total of 130 modules have been assembled in 79 weeks with a detector grade yield of $75 \%$, figure $7 \mathrm{a}$. In the OB production, over 2500 modules were assembled in 85 weeks with a detector grade yield of $84 \%$, figure $7 b$.
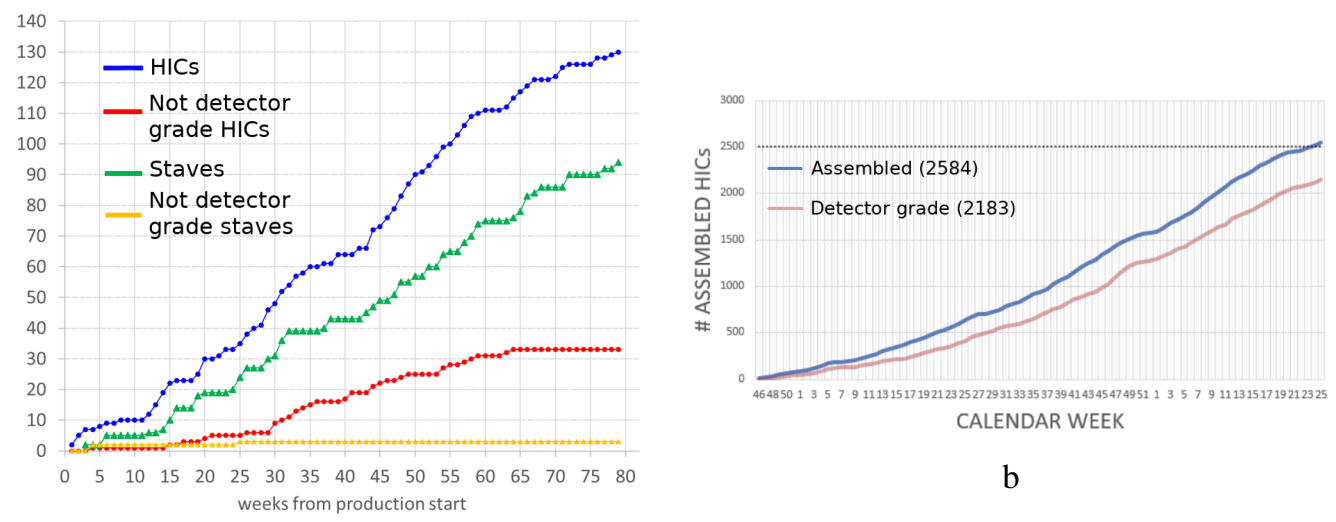

a

Figure 7: The production time lines for both the (a) IB modules and the (b) OB modules. 


\section{References}

[1] ALICE Collaboration, K. Aamodt et al., The ALICE experiment at the CERN LHC, JINST 3, 2008, S08002, DOI: 10.1088/1748-0221/3/08/s08002.

[2] ALICE Collaboration, B. Abelev et al., Upgrade of the ALICE Experiment: Letter Of Intent, J.Phys. G41, 2014, 087001, DOI: 10.1088/0954-3899/41/8/087001.

[3] ALICE Collaboration, B. Abelev et al., Technical Design Report for the Upgrade of the ALICE Inner Tracking System. J. Phys. G 41, 2014, 087002. DOI: 10.1088/0954-3899/41/8/087002.

[4] M. Mager, ALPIDE, the Monolithic Active Pixel Sensor for the ALICE ITS upgrade, Nucl. Instrum. Methods Phys. Res. A: 24, pp. 434-438, 2016. DOI: 10.1016/j.nima.2015.09.057. 